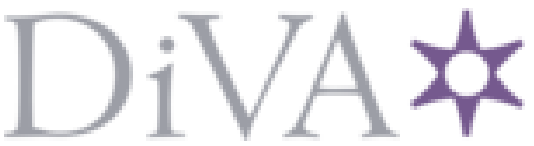

http://www.diva-portal.org

Preprint

This is the submitted version of a paper published in IEEE transactions on electromagnetic compatibility (Print).

Citation for the original published paper (version of record):

Malmström, J., Holter, H., Jonsson, L. (2017)

On Mutual Coupling and Coupling Paths between Antennas Using the Reaction Theorem

IEEE transactions on electromagnetic compatibility (Print)

Access to the published version may require subscription.

N.B. When citing this work, cite the original published paper.

Permanent link to this version:

http://urn.kb.se/resolve?urn=urn:nbn:se:kth:diva-215200 


\title{
On Mutual Coupling and Coupling Paths between Antennas Using the Reaction Theorem
}

\author{
Johan Malmström, Henrik Holter, B. L. G. Jonsson
}

\begin{abstract}
-
The reaction theorem is applied to antenna coupling problems. It is shown that the reaction theorem can be used to calculate the mutual impedance between antennas, when the electromagnetic fields are known on a plane that separates the antennas in two disjoint regions. We also show that coupling paths between the antennas can be visualized by using intermediate results from the reaction theorem. The coupling paths are visualized based on the fields generated by each of the two antennas, and only take into account the energy that is actually transferred between the antennas. The visualization of coupling paths is useful for understanding how the coupling between the antennas is distributed in space.
\end{abstract}

Index Terms-Electromagnetic compatibility, mutual impedance, mutual coupling, coupling paths.

\section{INTRODUCTION}

$\mathbf{T}$ HE MUTUAL COUPLING between antennas, and the related mutual impedance [1], is of major importance when installing antennas on platform [2]. A high mutual coupling means that much of the transmitted energy leaks into the other antenna, something that can severely degrade system performance [3].

The reaction [4]-[6] is a measure of the interaction between two sources, carried by their corresponding fields. The reaction theorem [4]-[6] relates the electromagnetic (EM) fields generated by the antennas with the mutual impedance between them. The reaction theorem has been used in several works for mutual impedance calculations, see e.g. [7]-[11].

Coupling paths are spatial descriptions of the mutual coupling, i.e. trajectories from the transmitting to the receiving equipment where the mutual coupling is established [12]. The coupling paths show potential ways where interference might come from and is an important tool in EMC studies. When designing complex systems, the coupling paths tend to be difficult to identify [13], [14]. Ray-tracing and other methods [15], briefly reviewed in [13], has been used to identify coupling paths in a systematic manner. These methods have reported drawbacks [13], e.g. they are only valid in the far-field region or involve complicated measurements [13].

Coupling paths should not be confused with Poynting's vector. The coupling paths contain only the part of the transmitted energy that reaches the receiver [12], [14]. Poynting's vector describe the power flow transmitted from one of the

Manuscript received October 20, 2017; revised Xxxx XX, 2017; accepted Xxxx XX 2017. Date of current version October 20, 2017.

J. Malmström and B. L. G. Jonsson are with the Electromagnetic Engineering Laboratory, KTH Royal Institute of Technology, Stockholm, Sweden. J. Malmström and H. Holter are with Saab Surveillance, Stockholm, Sweden.

The work was supported by Saab, ChaseOn/iAA and SSF/AM130011. (a)
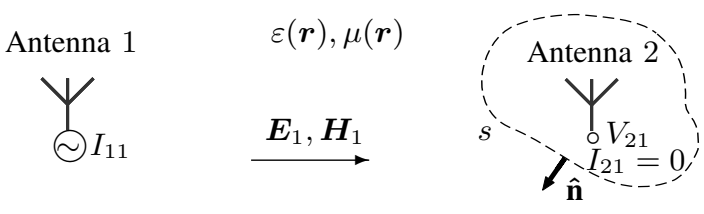

(b)
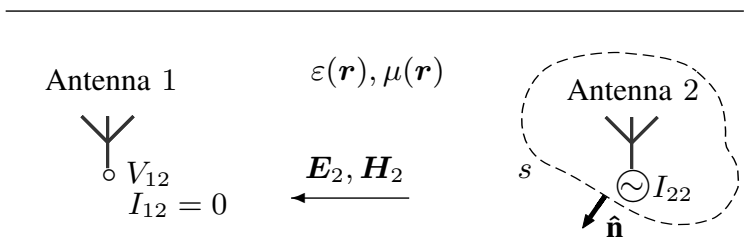

Fig. 1. The reaction theorem (2); (a) Antenna 1 transmits with a current $I_{11}$ while Antenna 2 is open circuit, and (b) Antenna 2 transmits with a current $I_{22}$ while Antenna 1 is open circuit. The media is the same in (a) and (b).

equipments or antennas involved [16], but it does not provide any information of the portion of that energy that actually reaches the receiving equipment.

\section{Mathematical Formulations}

\section{A. The Reaction Theorem}

The mutual impedance $Z_{21}$ between two antennas is defined as a ratio between the voltage $V_{21}$ and the current $I_{11}$ [1],

$$
Z_{21}=\left.\frac{V_{21}}{I_{11}}\right|_{I_{21}=0}
$$

assuming that the antenna terminal in the passive antenna is open circuit, as in Fig. 1(a).

The reaction theorem [4], [5], illustrated in Fig. 1(a)-(b), relates the mutual impedance $Z_{21}$ with the fields ${ }^{1} \boldsymbol{E}_{1}, \boldsymbol{H}_{1}$ generated by Antenna 1 and $\boldsymbol{E}_{2}, \boldsymbol{H}_{2}$ by Antenna 2,

$$
Z_{21}=\frac{-1}{I_{11} I_{22}} \oint_{s}\left(\boldsymbol{E}_{2} \times \boldsymbol{H}_{1}-\boldsymbol{E}_{1} \times \boldsymbol{H}_{2}\right) \cdot \hat{\mathbf{n}} \mathrm{d} S,
$$

where the closed integration surfaces $s$, with normal $\hat{\mathbf{n}}$, must separate the two antennas in two disjoint regions.

There is also a generalized reaction theorem in which the environment outside $s$ can be changed when the antenna enclosed by $s$ transmits [5]. The results in this letter apply also to the generalized reaction theorem.

\section{B. Mutual Impedance and Mutual Coupling}

The mutual impedance, $Z_{21}$, is related to the mutual coupling $S_{21}$ [1]. For well separated antennas, where the

\footnotetext{
${ }^{1}$ We use complex phasor notation with the time dependence $\mathrm{e}^{\mathrm{j} \omega t}$.
} 


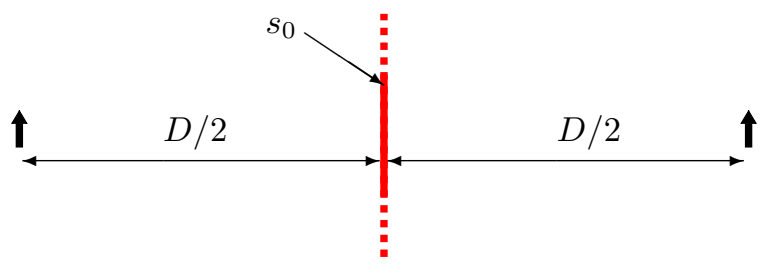

Fig. 2. Position of the separation plane $s_{0}$ relative to the two Hertzian dipoles.

mutual impedances $Z_{12}, Z_{21}$ are small compared to the self-impedances $Z_{11}, Z_{22}$ of the antennas, i.e. $\left|Z_{12} Z_{21}\right| \ll$ $\left|Z_{11} Z_{22}\right|$, we can write $S_{21}$ as a series expansion. By neglecting higher order terms, we can write an approximate relation

$$
S_{21} \approx Z_{21} \frac{2 Z_{0}}{\left(Z_{11}+Z_{0}\right)\left(Z_{22}+Z_{0}\right)} .
$$

Note that this approximation of $S_{21}$ is proportional to $Z_{21}$. Hence, for antennas with low mutual impedance $\left|Z_{12} Z_{21}\right| \ll$ $\left|Z_{11} Z_{22}\right|$, we can study the behavior of $Z_{21}$ instead of $S_{21}$. This is of interest in e.g. problems involving minimization of the mutual coupling between the antennas, see e.g. [11].

\section{The Integration Surface}

The shape of the integration surface $s$ in the reaction theorem (2) is arbitrary as long as it encloses one and only one of the antennas. In this paper we use a plane integration surface that separates the two antennas in two regions. We define the separation plane by first creating a Cartesian box with six faces $s=\cup\left(s_{0}, \ldots, s_{5}\right)$, each with side length $\ell$, that encloses one of the antennas. Let one of the faces, say $s_{0}$, be positioned between the antennas, as depicted in Fig. 2. We let the other five faces, $s_{1}, \ldots, s_{5}$, move away from the surrounded antenna, while the surface $s_{0}$ is fixed. The total area of the surface $s$ is $\mathcal{O}\left(\ell^{2}\right)$. Silver-Müller's radiation condition can be used to show that the reaction on the integration surfaces $s_{1}, \ldots, s_{5}$ goes to zero in the limit $\ell \rightarrow \infty$ [17]. Only the fields on the face $s_{0}$ will contribute to the reaction. Hence, the surface $s_{0}$ can be used as integration surface when calculating the mutual impedance with (2).

The plane integration surface is advantageous when visualizing the mutual impedance, as will be seen in Sec. III-C.

\section{Applications}

We apply the reaction theorem to EMC problems involving antennas on platforms. The reaction theorem is used on a separation plane to determine the mutual impedance; for antennas in free space (Sec. III-A) and for antennas installed on a conducting platform (Sec. III-B). It is shown that the integrand in the reaction integral can be used to visualizing coupling paths and to track coupling path trajectories (Sec. III-C).

\section{A. Hertzian Dipoles in Free Space}

For conceptual understanding, it is illustrative to study a very simple set-up; consider two identical Hertzian dipoles

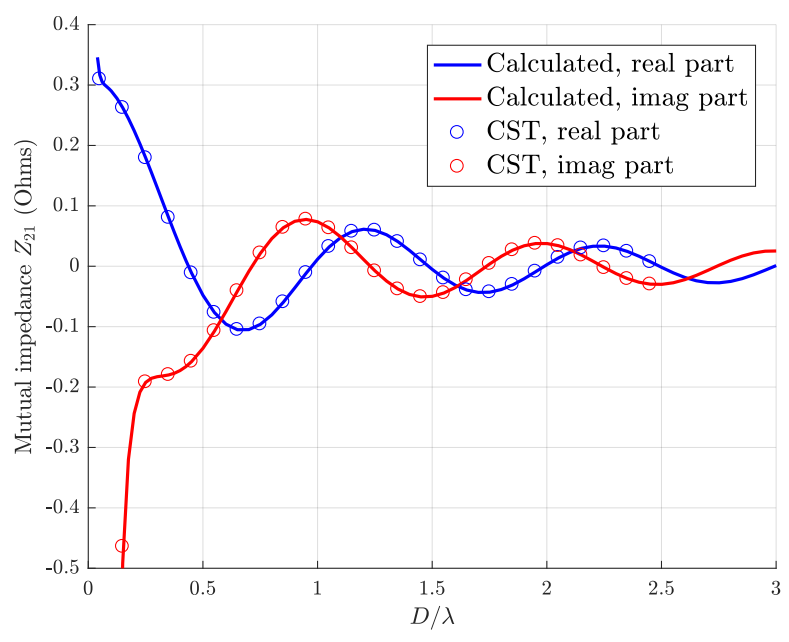

Fig. 3. Mutual impedance $Z_{21}$ between two Hertzian dipole placed side-byside (see Fig. 2) for varying separation distance $D / \lambda$ calculated with (2).

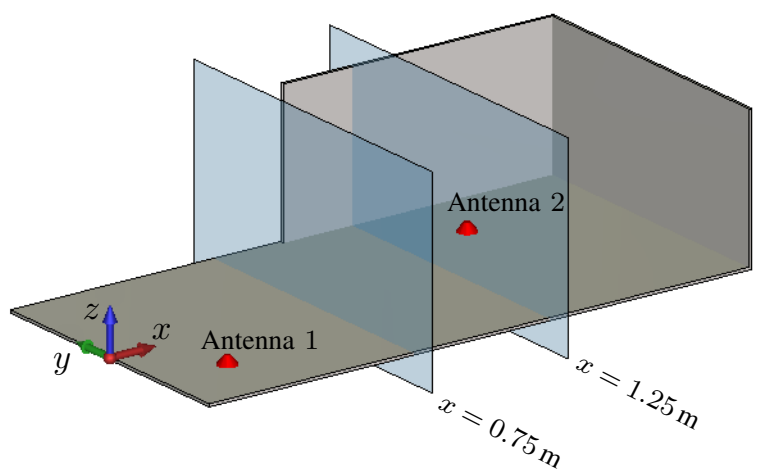

Fig. 4. Model of a platform with two antennas installed. The platform is $20 \lambda \times 10 \lambda \times 5 \lambda$ at the used frequency $3 \mathrm{GHz}$. The separation planes, $x=0.75 \mathrm{~m}$ and $x=1.25 \mathrm{~m}$ are also illustrated.

with length $20 \mathrm{~mm}$ at frequency $300 \mathrm{MHz}$ placed side-by-side in free space. The exact field expressions (see e.g. [6]) are used to calculate the fields on a separation plane $s_{0}$ placed symmetrically between the antennas, see Fig. 2 . The distance $D$ between the Hertzian dipoles is varied, $D=[\lambda / 20,3 \lambda]$. Scattering from Hertzian dipoles is very low and is neglected.

The resulting mutual impedance $Z_{21}$, calculated with the reaction theorem (2), is depicted in Fig. 3 together with the mutual impedance simulated with a full-wave solver [18]. The agreement between the two data sets is excellent and verifies that a separation plane can be used as integration surface with the reaction theorem (2) in this case. The result in Fig. 3 also agrees with previously reported results [19]. The calculation generalizes to any type of antenna, and requires only that the fields on the separation plane are known.

\section{B. Antennas on Platforms}

A more realistic problem is to analyze antennas on a common platform. A simplified platform is used to demonstrate the reaction theorem with a separation plane. The $2 \mathrm{~m} \mathrm{x}$ $1 \mathrm{~m} \times 0.5 \mathrm{~m}$ platform, depicted in Fig. 4, consists of a PEC plate in the $x y$ plane, with two $1 \mathrm{~m} \times 0.5 \mathrm{~m}$ walls 


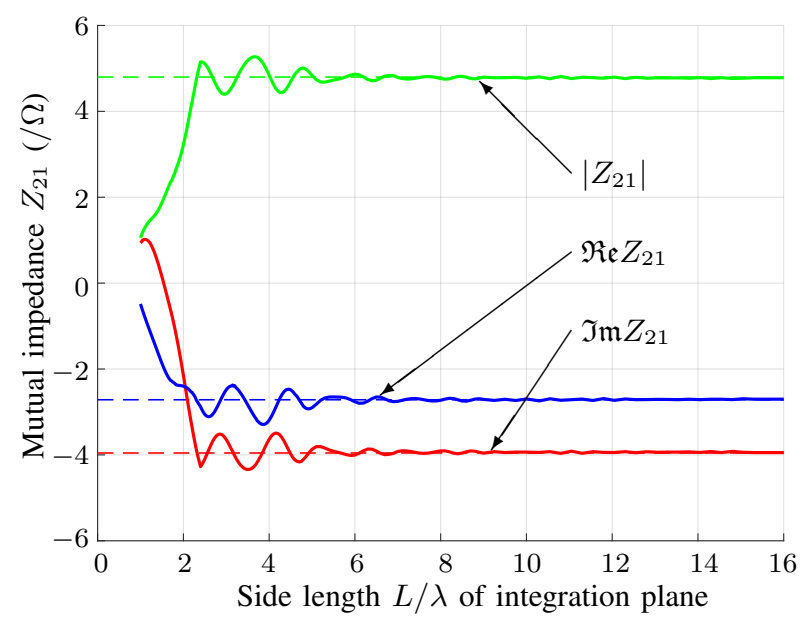

Fig. 5. Mutual impedance $Z_{21}$ between the two fixed antennas depicted in Fig. 4 at $3 \mathrm{GHz}$ calculated with the reaction theorem (2) for varying sizes of integration plane $s_{0}$ (solid lines) and with a full-wave solver (dashed lines).

in the $x z$ and $y z$ planes, respectively. The thickness of the plate and the walls are $10 \mathrm{~mm}$. Two monopole antennas are mounted on the platform at $\boldsymbol{r}_{1}=(0.25 \mathrm{~m},-0.25 \mathrm{~m}, 0)$ and $\boldsymbol{r}_{2}=(1.5 \mathrm{~m}, 0.25 \mathrm{~m}, 0)$. The antennas are excited at $3 \mathrm{GHz}$, which is close to their resonance frequency.

Ideally, the separation plane $s_{0}$ should be infinitely large, but for practical reasons, we truncate it when the integrand is small. We let $s_{0}$ be a square with area $L^{2}$, with its center at $\boldsymbol{r}_{0}=(0.75 \mathrm{~m}, 0,0.25 \mathrm{~m})$. That is $0.50 \mathrm{~m}$ from Antenna 1 and $0.75 \mathrm{~m}$ from Antenna 2 .

The mutual impedance between the antennas calculated with the reaction (2) is depicted in Fig. 5 for varying size $L / \lambda$ of the integration plane $s_{0}$. For comparison, we also plot the mutual impedance $Z_{21}$ calculated with a commercial EM simulation software [18] as reference. We see that the mutual impedance oscillates for small integration planes. When the integration plane becomes larger, the calculated $Z_{21}$ converges toward the reference value and confirms that the reaction theorem can be used to calculate $Z_{21}$ between antennas on a common platform.

\section{Visualizing Coupling Paths}

From the reaction theorem (2), we know that the impedance $Z_{21}$ can be calculated as an integral over the separation plane. Based on the integrand in (2), we define the vector impedance density $^{2} \varrho_{21}(\boldsymbol{r})$ as,

$$
\varrho_{21}(\boldsymbol{r})=-\frac{\boldsymbol{E}_{2}(\boldsymbol{r}) \times \boldsymbol{H}_{1}(\boldsymbol{r})-\boldsymbol{E}_{1}(\boldsymbol{r}) \times \boldsymbol{H}_{2}(\boldsymbol{r})}{I_{11} I_{22}} .
$$

The direction of $\varrho_{21}(\boldsymbol{r})$ describes trajectories between the two antennas. The real part of the vector impedance density $\mathfrak{R e} \varrho_{21}(\boldsymbol{r})$ is depicted in Fig. $6^{3}$ for the structure in Fig. 4 and

\footnotetext{
${ }^{2}$ We note that the numerator in (4) has dimension power per area, which is identical to the dimension of Poynting's vector.

${ }^{3}$ Fig. 6 illustrate the instantaneous vector impedance density $\varrho_{21}(\boldsymbol{r})$, as defined in (4). A time-averaged vector impedance density $-\frac{1}{2} \mathfrak{R e}\left(\boldsymbol{E}_{2}(\boldsymbol{r}) \times\right.$ $\left.\boldsymbol{H}_{1}^{*}(\boldsymbol{r})-\boldsymbol{E}_{1}(\boldsymbol{r}) \times \boldsymbol{H}_{2}^{*}(\boldsymbol{r})\right) / I_{11} I_{22}$ is also useful for visualization, especially for identifying radiated and electromagnetic guided coupling [14].
}

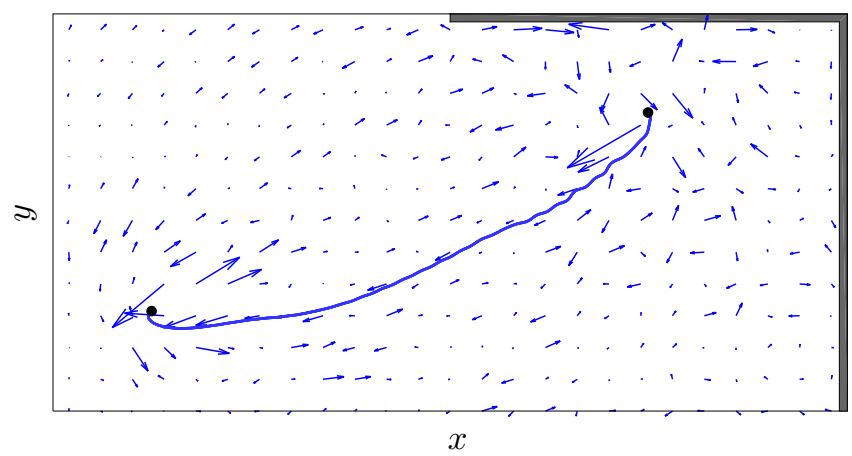

Fig. 6. The vector arrows illustrate $\mathfrak{R e} \varrho_{21}(\boldsymbol{r})$ between the two antennas in Fig. 4 for $z=10 \mathrm{~mm}$. One coupling path trajectory (solid line) is tracked with $\mathfrak{R e} \varrho_{21}(\boldsymbol{r})$.

with $\boldsymbol{r}=\left(x, y, z_{0}\right), z_{0}=10 \mathrm{~mm}$ together with one coupling path trajectory tracked with $\mathfrak{R e} \varrho_{21}(\boldsymbol{r})$.

We also define the projected impedance density $\varrho_{21}(\boldsymbol{r})$ as,

$\varrho_{21}(\boldsymbol{r})=-\frac{\left(\boldsymbol{E}_{2}(\boldsymbol{r}) \times \boldsymbol{H}_{1}(\boldsymbol{r})-\boldsymbol{E}_{1}(\boldsymbol{r}) \times \boldsymbol{H}_{2}(\boldsymbol{r})\right) \cdot \hat{\mathbf{n}}(\boldsymbol{r})}{I_{11} I_{22}}$.

The projected impedance density $\varrho_{21}(\boldsymbol{r})$ depends on the position $r$ and the surface normal $\hat{\mathbf{n}}$. It describes how the mutual impedance is distributed over points $r$ on the integration surface e.g. a separation plane with a constant normal $\hat{\mathbf{n}}(\boldsymbol{r})=\hat{\mathbf{n}}_{0}$. The value $\varrho_{21}(\boldsymbol{r})$ in a certain point $\boldsymbol{r}$ is a complex number and will either add or subtract to the mutual impedance $Z_{21}$ between the antennas.

Note that integrating $\varrho_{21}(\boldsymbol{r})$ over the separation plane results in the mutual impedance $Z_{21}$. Based on this observation, we define a normalized impedance density as,

$$
\widetilde{\varrho}_{21}(\boldsymbol{r})=\frac{\varrho_{21}(\boldsymbol{r})}{Z_{21}} \text {. }
$$

The normalized impedance density $\widetilde{\varrho}_{21}(\boldsymbol{r})$ integrates over the separation plane $s_{0}$ to the real value 1 .

Fig. 7 depicts the real part of the normalized impedance density $\mathfrak{R e} \widetilde{\varrho}_{21}(\boldsymbol{r})$ for fixed antenna positions and two different positions of the separation plane, $x=0.75 \mathrm{~m}$ and $x=1.25 \mathrm{~m}$. To get a full picture of the normalized impedance density ${ }^{4}$, the imaginary part, $\mathfrak{I m} \widetilde{\varrho}_{21}(\boldsymbol{r})$, on the same separation planes $s_{0}$ is depicted in Fig. 8. Because of the normalization in (6), the imaginary part $\mathfrak{I m} \widetilde{\varrho}_{21}(\boldsymbol{r})$ integrates to zero over $s_{0}$.

From the normalized impedance density $\widetilde{\varrho}_{21}(\boldsymbol{r})$ depicted in Fig. 7-8, strong coupling paths can be identified. In initial studies, we have decreased the mutual impedance by blocking identified strong coupling paths with absorbing material.

Note that $\varrho_{21}(\boldsymbol{r})$ does not depend on the integration surface. Hence, $\varrho_{21}(\boldsymbol{r})$ can be calculated for any point in space where the fields are known. Opposite, $\varrho_{21}(\boldsymbol{r})$ and $\widetilde{\varrho}_{21}(\boldsymbol{r})$ require that an integration surface with a known normal $\hat{\mathbf{n}}_{2}(\boldsymbol{r})$ is defined.

\footnotetext{
${ }^{4} \mathrm{We}$ want to distinguish between positive and negative parts of $\widetilde{\varrho}_{21}(\boldsymbol{r})$, therefore the magnitude $\left|\widetilde{\varrho}_{21}(\boldsymbol{r})\right|$ does not provide all information of interest.
} 


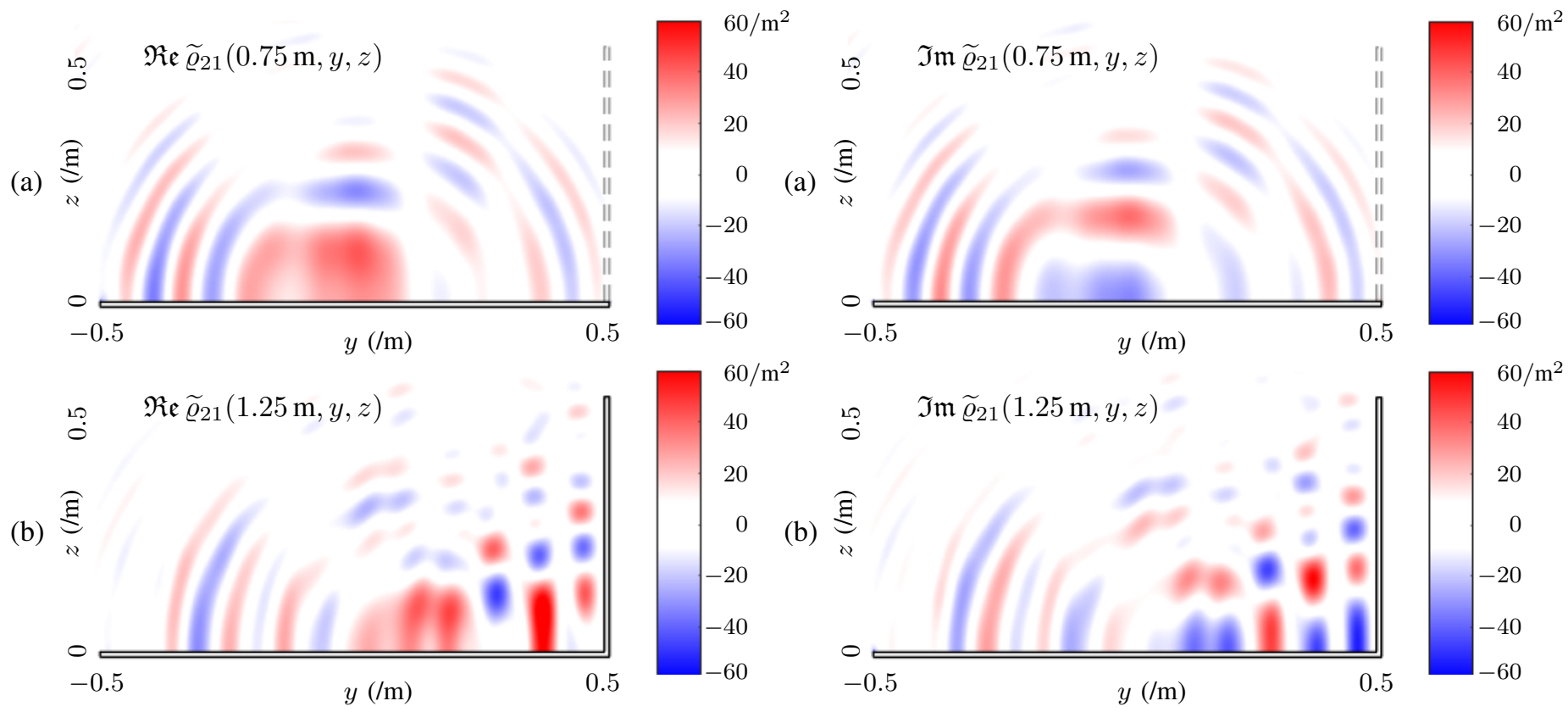

Fig. 7. Coupling paths illustrated by the normalized impedance density $\mathfrak{R e} \widetilde{\varrho}_{21}(\boldsymbol{r})$ in $1 / \mathrm{m}^{2}$ on separation planes at (a) $x=0.75 \mathrm{~m}$ and (b) $x=1.25 \mathrm{~m}$.

\section{Discussion AND CONCLUSIONS}

We have applied the reaction theorem to calculate the mutual impedance between antennas installed on platforms based on the EM fields generated by the antennas on a separation plane.

Based on the reaction theorem, we have defined a vector impedance density (4), a projected impedance density (5), and a normalized impedance density (6) that illustrate the distribution of the coupling paths between the antennas. We have used the vector impedance density to find coupling path trajectories, in the same way as Poynting's vector is used in [14]. Strong coupling paths has been identified by inspecting the normalized impedance density over the separation plane. In initial tests, we have decreased the mutual impedance by material changes in regions with identified high normalized impedance densities.

The input data for calculating the mutual impedance or normalized impedance density are EM fields generated by each of the two antennas. This data can be calculated and exported by commercial EM simulation software. Hence, the calculations demonstrated can be applied as a post-processing step after an EM simulation.

\section{REFERENCES}

[1] D. M. Pozar, Microwave Engineering, 4th ed. John Wiley \& Sons, 2005.

[2] T. M. Macnamara, Introduction to Antenna Placement and Installation. John Wiley \& Sons, 2010.

[3] E. M. Koper, W. D. Wood, and S. W. Schneider, "Aircraft antenna coupling minimization using genetic algorithms and approximations," IEEE Trans. Aerosp. Electron. Syst., vol. 40, no. 2, pp. 742-751, 2004.

Fig. 8. Coupling paths illustrated by the normalized impedance density $\mathfrak{I m} \widetilde{\varrho}_{21}(\boldsymbol{r})$ in $1 / \mathrm{m}^{2}$ on separation planes at (a) $x=0.75 \mathrm{~m}$ and (b) $x=1.25 \mathrm{~m}$.

[4] V. H. Rumsey, "Reaction concept in electromagnetic theory," Phys. Rev., vol. 94, no. 6, pp. 1483-1491, 1954.

[5] J. H. Richmond, "A reaction theorem and its application to antenna impedance calculations," Antennas Propagation, IRE Trans., vol. 9, no. 6, pp. 515-520, 1961.

[6] C. A. Balanis, Antenna Theory Analysis and Design, 3rd ed. John Wiley \& Sons, 2005.

[7] Wang H. et. al, "Estimating radio-frequency interference to an antenna due to near-field coupling using decomposition method based on reciprocity," IEEE Trans. Electromagn. Compat., vol. 55, no. 6, pp. 1125$1131,2013$.

[8] Foged, L. J. et al., "Innovative representation of antenna measured sources for numerical simulations," in IEEE Antennas Propag. Soc. AP-S Int. Symp., 2014, pp. 2014-2015.

[9] L. Li, J. Pan, C. Hwang, and J. Fan, "Radiation Noise Source Modeling and Application in Near-Field Coupling Estimation," IEEE Trans. Electromagn. Compat., vol. 58, no. 4, pp. 1314-1321, 2016.

[10] C. Craeye, "On the transmittance between OAM antennas," IEEE Trans. Antennas Propag., vol. 64, no. 1, pp. 336-339, 2016.

[11] J. Malmström and B. L. G. Jonsson, "An Effective Method for Antenna Placement on Platforms Based on the Reaction Theorem," in 2017 Int. Conf. on Electromagn. in Adv. Appl. (ICEAA), 2017.

[12] C. R. Paul, Introduction to Electromagnetic Compatibility, 2nd ed. John Wiley \& Sons, 2006.

[13] H. Li, V. V. Khilkevich, and D. Pommerenke, "Identification and visualization of coupling paths - Part I: Energy parcel and its trajectory," IEEE Trans. Electromagn. Compat., vol. 56, no. 3, pp. 622-629, 2014

[14] — , "Identification and Visualization of Coupling Paths - Part II: Practical Application," IEEE Trans. Electromagn. Compat., vol. 56, no. 3, pp. 630-637, 2014.

[15] U. Paoletti, "On the Noise Path Determination With the Noise Source Modulation Technique," IEEE Trans. Electromagn. Compat., vol. 59, no. 5, pp. 1400-1407, 2017.

[16] J. D. Jackson, Classical Electrodynamics, 3rd ed. John Wiley \& Sons, 1998.

[17] J. Malmström, "On the Reaction Theorem for Scattered Fields," submitted for publication, 2017.

[18] CST, "Microwave Studio," 2017.

[19] L. D. Bamford, P. S. Hall, and A. Fray, "Calculation of antenna mutual coupling from radiated far fields," in Comput. Electromagn. 1994., Second Int. Conf., 1994, pp. 263-266. 